\title{
Intelligent Extension-Models for Non-Circular Curves and Their Applications Based on Trend Extrapolation
}

\author{
Youyu $\operatorname{Liu}^{1,2 *}$ \\ ${ }^{1}$ School of Mechanical and Automotive Engineering, Anhui Polytechnic University, \\ Wuhu 241000, China \\ ${ }^{2}$ Institute of CIMS, Hefei University of Technology, Hefei 230009, China \\ liuyoyu1@163.com
}

\begin{abstract}
Up to now, plenty of CAD softwares have no function of extension for non-circular curves. Intelligent extension-technology for non-circular curves was studied in this article based on a principle of trend extrapolation. Four kinds of intelligent extension-models were built. These models include quadratic extension-model, cubic extension-model, exponential extensionmodel, and Gompertz extension-model. Moreover, some methods identifying and choosing for extension-models were discussed, respectively. Several applications of intelligent extension for non-circular curves were offered, which shows us that some curves can be extended for a long distance accurately while they are consistent with the type of extension-models; on the other hand, extension-models built can be available for approximate extension for a short distance while the shapes of non-circular curves are arbitrary. The theories in this paper, as core technologies and key generic problems, can be used for existing CAD softwares to upgrade or develop secondarily.
\end{abstract}

Keywords: Intelligent extension-models, Models identifying and choosing, Non-circular curves, Trend extrapolation.

\section{Introduction}

Modern CAD softwares have been developing greatly. Plenty of 2-D or 3-D softwares, such as AutoCAD, CAXA, Pro/E, UG and CATIA, have some characteristics of friendly interface and powerful functions. All of softwares mentioned have the basic function of extension. They can extend a line or arc or ellipse to a specified object without changing original properties (line or arc or ellipse). However, the above softwares cannot extend a noncircular curve (except ellipse). While calling extension, some softwares (e.g., AutoCAD) cannot do anything; some softwares (e.g., CAXA) extend it as a line regardless of its original properties, which will lead to significant errors. In project charting or practical 3-D modeling, we need to extend some non-circular curves to some specified objects frequently.

Trend extrapolation [1] is a prediction technology used for scientific and technological progress, economic and social development, which is of great importance. The basic assumption is that the future is a continuing process based on past and present. Trend extrapolation is currently used for intelligence research, such as analysis and extrapolation of stock market indexes [2], tobacco consumption trends and their relationship to lung cancer mortality [3], trends in atmosphere, sea ice and ocean [4]. For the sake of the similarities between curve extension and trend extrapolation in intelligence research, we had studied an intelligent extension-technology for non-circular curves under polar coordinates [5], in which the study is rather superficial and less practical. In this article, to solve such common and core

*Corresponding Author 
problems in CAD softwares, a trend extrapolation principle is imported and an intelligent extension-technology for non-circular curves is also discussed comprehensively under rectangular coordinates, which will be more applicable. Moreover, its steps and methods are offered with examples. We will also discuss the accuracy of the extension-models, and point out their using scopes and conditions.

\section{Intelligent Extension-Models}

\subsection{Multinomial Extension-Models}

Except for linear and arc, almost all curves to be extended for CAD softwares are generally splines, of which the shapes are controlled by several feature points [6]. Using trend extrapolation, we can choose a suitable curve-model for a non-circular curve according to the changing trend of the feature points, and extend it following the trend under the character of continuity. It is obvious that different feature points, determining different curves, should adopt different extension-models. Several typical extension-models have been developed in this article, which can be chosen according to the feature of original curves.

2.1.1 Quadratic Extension-Model: Discrete quadratic curve equation [7] is as follows.

$$
y_{i}=a x_{i}^{2}+b x_{i}+c \quad(i=1,2, \ldots, m)
$$

Where $y_{i}$ is coordinating values $\boldsymbol{y}$ of points $i ; x_{i}$ is coordinating values $\boldsymbol{x}$ of points $i$; $i=1,2, \ldots, m ; a, b$, and $c$ are constant coefficients.

When $x_{i}$ constitutes an arithmetic progression from some feature points, second-order difference $\nabla^{2} y_{i}[8]$ of sequences $\left\{y_{i}\right\}$ from the feature points is shown in Eq. (2). It is a constant.

$$
\nabla^{2} y_{i}=y_{i+2}-2 y_{i+1}+y_{i}=2 a
$$

Consequently, while the second-order difference of a discrete curve is near to constant, a quadratic extension-model, as shown in Eq. (3), can be adopted.

$$
\hat{y}_{i}=\hat{a} \hat{x}_{i}^{2}+\hat{b} \hat{x}_{i}+\hat{c} \quad(i=m+1, m+2, \ldots, n)
$$

Where $\hat{y}_{i}$ is coordinating value $\boldsymbol{y}$ of point $i$ extended; $x_{i}$ is coordinating value $\boldsymbol{x}$ of points $i$ extended; $i=m+1, m+2, \ldots, n ; \hat{a}, \hat{b}$ and $\hat{c}$ are model parameters.

The undetermined parameters can be determined by the Least Square Method [9]. From Eq. (3) we can draw Eq. (4) and Eq. (5).

$$
\begin{gathered}
e_{i}=y_{i}-\hat{y}_{i}=y_{i}-\hat{a} x_{i}^{2}-\hat{b} x_{i}-\hat{c} \\
Q=\sum_{i=1}^{m} e_{i}^{2}=\sum_{i=1}^{m}\left(y_{i}-\hat{a} x_{i}^{2}-\hat{b} x_{i}-\hat{c}\right)^{2}
\end{gathered}
$$

Where $y_{i}$ is coordinating value $\boldsymbol{y}$ of pre-existing points $i ; e_{i}$ is dispersion of points $i ; Q$ is sum of deviation square.

We can gain $\partial Q / \partial \hat{a}, \partial Q / \partial \hat{b}$ and $\partial Q / \partial \hat{c}$ from Eq. (5), and let all of them equal to zero. Thus, Eqs. (6) with independent variables $\hat{a}, \hat{b}$ and $\hat{c}$ can be obtained. 


$$
\left\{\begin{array}{l}
\sum_{i=1}^{m} y_{i}=\hat{a} \sum_{i=1}^{m} x_{i}^{2}+\hat{b} \sum_{i=1}^{m} x_{i}+\hat{c} n \\
\sum_{i=1}^{m} x_{i} y_{i}=\hat{a} \sum_{i=1}^{m} x_{i}^{3}+\hat{b} \sum_{i=1}^{m} x_{i}^{2}+\hat{c} \sum_{i=1}^{m} x_{i} \\
\sum_{i=1}^{m} x_{i}^{2} y_{i}=\hat{a} \sum_{i=1}^{m} x_{i}^{4}+\hat{b} \sum_{i=1}^{m} x_{i}^{3}+\hat{c} \sum_{i=1}^{m} x_{i}^{2}
\end{array}\right.
$$

While number of sequence $x_{i}$ is an odd, and its middle term $x_{(m+1) / 2}$ equals to zero, $\sum_{i=1}^{m} x_{i}=0$, and $\sum_{i=1}^{m} x_{i}^{3}=0$. Then, Eqs. (6) can be simplified as follows.

$$
\left\{\begin{array}{l}
\sum_{i=1}^{m} y_{i}=\hat{a} \sum_{i=1}^{m} x_{i}^{2}+\hat{c} n \\
\sum_{i=1}^{m} x_{i} y_{i}=\hat{b} \sum_{i=1}^{m} x_{i}^{2} \\
\sum_{i=1}^{m} x_{i}^{2} y_{i}=\hat{a} \sum_{i=1}^{m} x_{i}^{4}+\hat{c} \sum_{i=1}^{m} x_{i}^{2}
\end{array}\right.
$$

Solving the equations above,

$$
\left\{\begin{array}{l}
\hat{a}=\frac{m \sum_{i=1}^{m} x_{i}^{2} y_{i}-\sum_{i=1}^{m} x_{i}^{2} \sum_{i=1}^{m} y_{i}}{m \sum_{i=1}^{m} x_{i}^{4}-\left(\sum_{i=1}^{m} x_{i}^{2}\right)^{2}} \\
\hat{b}=\frac{\sum_{i=1}^{m} x_{i} y_{i}}{\sum_{i=1}^{m} x_{i}^{2}} \\
\hat{c}=\frac{\sum_{i=1}^{m} x_{i}^{4} \sum_{i=1}^{m} y_{i}-\sum_{i=1}^{m} x_{i}^{2} \sum_{i=1}^{m} x_{i}^{2} y_{i}}{n \sum_{i=1}^{m} x_{i}^{4}-\left(\sum_{i=1}^{m} x_{i}^{2}\right)^{2}}
\end{array}\right.
$$

The undetermined parameters can be calculated by Eqs. (8) with the feature points. These parameters can be substituted in Eq. (3), and then an extension-model is built.

2.1.2. Cubic Extension-Model: Discrete cubic curve equation [7] is as follows.

$$
y_{i}=a x_{i}^{3}+b x_{i}^{2}+c x_{i}+d \quad(i=1,2, \ldots, m)
$$

Where $d$ is a constant coefficient; implications of other parameters are the same as above. When $x_{i}$ constitutes an arithmetic progression from some feature points, the third-order difference $\nabla^{3} y_{i}$ [10] of sequences $\left\{y_{i}\right\}$ from the feature points is shown in Eq. (10). It is a constant.

$$
\nabla^{3} y_{i}=y_{i+3}-3 y_{i+2}+3 y_{i+1}-y_{i}=6 a
$$

Consequently, while the third-order difference of a discrete curve is near to constant, a cubic extension-model, as shown in Eq. (11), can be adopted.

$$
\hat{y}_{i}=\hat{a} x_{i}^{3}+\hat{b} x_{i}^{2}+\hat{c} x_{i}+\hat{d} \quad(i=m+1, m+2, \ldots, n)
$$

Where $\hat{y}_{i}$ is coordinating value $\boldsymbol{y}$ of points $i$ extended; $x_{i}$ is coordinating value $\boldsymbol{x}$ of points $i$ extended; $i=m+1, m+2, \ldots, n ; \hat{d}$ is model parameter. 
Like the quadratic extension-model, if number of the sequence $x_{i}$ is an odd, the undetermined parameters in Eq. (11) can be determined by the Least Square Method.

$$
\left\{\begin{array}{l}
\mid \hat{a}=\frac{\sum_{i=1}^{m} x_{i}^{4} \sum_{i=1}^{m} y_{i}-\sum_{i=1}^{m} x_{i}^{2} \sum_{i=1}^{m} x_{i}^{2} y_{i}}{m \sum_{i=1}^{m} x_{i}^{4}-\left(\sum_{i=1}^{m} x_{i}^{2}\right)^{2}} \\
\hat{b}=\frac{\sum_{i=1}^{m} x_{i}^{4} \sum_{i=1}^{m} x_{i}^{3} y_{i}-\sum_{i=1}^{m} x_{i}^{6} \sum_{i=1}^{m} x_{i} y_{i}}{\left(\sum_{i=1}^{m} x_{i}^{4}\right)^{2}-\sum_{i=1}^{m} x_{i}^{6} \sum_{i=1}^{m} x_{i}^{2}} \\
\hat{c}=\frac{m \sum_{i=1}^{m} x_{i}^{2} y_{i}-\sum_{i=1}^{m} x_{i}^{2} \sum_{i=1}^{m} y_{i}}{m \sum_{i=1}^{m} x_{i}^{4}-\left(\sum_{i=1}^{m} x_{i}^{2}\right)^{2}}
\end{array} \mid \begin{array}{l}
\hat{d}=\frac{\sum_{i=1}^{m} x_{i}^{4} \sum_{i=1}^{m} x_{i} y_{i}-\sum_{i=1}^{m} x_{i}^{2} \sum_{i=1}^{m} x_{i}^{3} y_{i}}{\left(\sum_{i=1}^{m} x_{i}^{4}\right)^{2}-\sum_{i=1}^{m} x_{i}^{2} \sum_{i=1}^{m} x_{i}^{6}}
\end{array}\right.
$$

The undetermined parameters can be calculated by Eqs. (12) with the feature points. These parameters can be substituted in Eq. (11). Then an extension-model is to be built.

\subsection{Exponential Extension-Model}

Discrete exponential curve equation [11] is as follows.

$$
y_{i}=b e^{a x_{i}} \quad(i=1,2, \ldots, m)
$$

Where $a$ and $b$ are constant coefficients; $i=1,2, \ldots, m$.

When $x_{i}$ constitutes an arithmetic progression from some feature points, first-order difference $\nabla \ln y_{i}[12]$ of logarithmic sequences $\left\{\ln y_{i}\right\}$ from the feature points is shown in Eq. (14). It is a constant.

$$
\nabla \ln y_{i}=a
$$

Consequently, while first-order difference of logarithmic sequences of a discrete curve is near to constant, an exponential extension-model, as shown in Eq. (15), can be adopted.

$$
\hat{y}_{i}=\hat{b} e^{\hat{a} x_{i}}
$$

Eq. (15) can be transformed into Eq. (16) by taking its log.

$$
\ln \hat{y}_{i}=\hat{a} x_{i}+\ln \hat{b}
$$

Let $\hat{y}_{i}^{\prime}=\ln \hat{y}_{i}$, and $\hat{b}^{\prime}=\ln \hat{b}$, and then Eq. (16) can be transformed into Eq. (17).

$$
\hat{y}_{i}^{\prime}=\hat{a} x_{i}+\hat{b}^{\prime}
$$

Therefore, the exponential extension-model (15) can be transformed into a linear extension-model (17).

The undetermined parameters can be determined by the Least Square Method. From Eq. (17), we can infer the following.

$$
e_{i}=y_{i}^{\prime}-\hat{y}_{i}^{\prime}=y_{i}^{\prime}-\hat{a} x_{i}-\hat{b}^{\prime}
$$




$$
Q=\sum_{i=1}^{m} e_{i}^{2}=\sum_{i=1}^{m}\left(y_{i}^{\prime}-\hat{a}^{\prime}-\hat{b} x_{i}\right)^{2}
$$

We can gain $\partial Q / \partial \hat{a}$ and $\partial Q / \partial \hat{b}^{\prime}$ from Eq. (19), and let all of them equal to zero. Thus, Eqs. (20) with independent variables $\hat{a}$ and $\hat{b}^{\prime}$ can be obtained.

$$
\left\{\begin{array}{l}
\sum_{i=1}^{m} y_{i}^{\prime}=m \hat{b}+\hat{a}^{\prime} \sum_{i=1}^{m} x_{i} \\
\sum_{i=1}^{m} x_{i} y_{i}^{\prime}=\hat{b} \sum_{i=1}^{m} x_{i}+\hat{a}^{\prime} \sum_{i=1}^{m} x_{i}^{2}
\end{array}\right.
$$

Solving the equations above,

$$
\left\{\begin{array}{l}
\hat{a}^{\prime}=\frac{m \sum_{i=1}^{m} x_{i} y_{i}^{\prime}-\sum_{i=1}^{m} x_{i} \sum_{i=1}^{m} y_{i}^{\prime}}{m \sum_{i=1}^{m} x_{i}^{2}-\left(\sum_{i=1}^{m} x_{i}\right)^{2}} \\
\hat{b}=\frac{\sum_{i=1}^{m} y_{i}^{\prime} \sum_{i=1}^{m} x_{i}^{2}-\sum_{i=1}^{m} x_{i} y_{i}^{\prime} \sum_{i=1}^{m} x_{i}}{m \sum_{i=1}^{m} x_{i}^{2}-\left(\sum_{i=1}^{m} x_{i}\right)^{2}}
\end{array}\right.
$$

For $\hat{y}_{i}^{\prime}=\ln \hat{y}_{i}$, and $\hat{b}^{\prime}=\ln \hat{b}$, Eqs. (21) can be transformed into Eqs. (22).

$$
\left\{\begin{array}{l}
\hat{a}=\exp \left(\frac{m \sum_{i=1}^{m} x_{i} \ln y_{i}-\sum_{i=1}^{m} x_{i} \sum_{i=1}^{m} \ln y_{i}}{m \sum_{i=1}^{m} x_{i}{ }^{2}-\left(\sum_{i=1}^{m} x_{i}\right)^{2}}\right) \\
\hat{b}=\frac{\sum_{i=1}^{m} \ln y_{i} \sum_{i=1}^{m} x_{i}{ }^{2}-\sum_{i=1}^{m} x_{i} \ln y_{i} \sum_{i=1}^{m} x_{i}}{m \sum_{i=1}^{m} x_{i}{ }^{2}-\left(\sum_{i=1}^{m} x_{i}\right)^{2}}
\end{array}\right.
$$

The undetermined parameters $\hat{a}$ and $\hat{b}$ can be calculated by Eqs. (22) with the feature points. These parameters can be substituted in Eq. (15), and then an extension-model is built.

\subsection{Gompertz Extension-Model}

Discrete Gompertz curve equation [13] is as follows.

$$
y_{i}=k a^{b^{x_{i}}}
$$

Where $k$ is a constant coefficient; $i=1,2, \ldots, m$; implications of other parameters are the same as above.

Eq. (23) can be transformed into Eq. (24).

$$
\ln y_{i}=b^{x_{i}} \ln a+\ln k
$$

When $x_{i}$ constitutes an arithmetic progression from some feature points, chain growth factor about first-order difference $\nabla \ln y_{i}$ [12] of logarithmic sequences $\left\{\ln y_{i}\right\}$ from the feature points is shown in Eq. (25). It is a constant.

$$
\frac{\nabla \ln y_{i+1}-\nabla \ln y_{i}}{\nabla \ln y_{i}}=b-1
$$

Consequently, as chain growth factor about first-order difference of logarithmic sequences of a discrete curve is near to constant, a Gompertz extension-model shown in Eq. (26) can be adopted.

$$
\hat{y}_{i}=\hat{k} \hat{a}^{\hat{b}^{x_{i}}}
$$


We can select some feature points $M=3 \mathrm{~m}$, which can be divided into three groups. Every group includes $m$ feature points. If the number of feature points is not equal to $3 m$ at first, we can abandon some points farther away from the location to be extended. These feature points can be substituted in Eqs. (27).

$$
\left\{\begin{array}{l}
\sum I_{1}=\ln y_{0}+\ln y_{1}+\ln y_{2} \\
\sum I_{2}=\ln y_{3}+\ln y_{4}+\ln y_{5} \\
\sum I_{3}=\ln y_{6}+\ln y_{7}+\ln y_{8}
\end{array}\right.
$$

Substituting the result from Eqs. (27) in Eqs. (28).

$$
\left\{\begin{array}{l}
\hat{b}^{n}=\frac{\sum I_{3}-\sum I_{2}}{\sum I_{2}-\sum I_{1}} \\
\ln \hat{a}=\frac{\left(\sum I_{2}-\sum I_{1}\right)(\hat{b}-1)}{\left(\hat{b}^{n}-1\right)^{2}} \\
\ln \hat{k}=\frac{1}{n}\left(\sum 1-\frac{\hat{b}^{n}-1}{\hat{b}-1} \ln \hat{a}\right)
\end{array}\right.
$$

The undetermined parameters $\hat{a}, \hat{k}$ and $\hat{b}$ can be calculated by Eqs. (28) with the feature points. These parameters can be substituted in Eq. (26), and then an extensionmodel is built.

\section{Applications of Intelligent Extension for Curves}

In order to evaluate the effectiveness of the intelligent extension-models, we extend some curves with given equations by selecting appropriate models from the intelligent extension-models above. Thus, we can analyze the tolerance of those extended curves with their theoretical curves.

As shown in Figure 1, for four kinds of extension-model, coordinate values $x_{i}$ of feature points on curves to be extended must be able to constitute an arithmetic progression. If not, we should fit the feature points $\left\{x_{i}, y_{i}\right\}$ with a spline curve [14], and then reselect several new feature points from the curve to make $x_{i}$ be an arithmetic progression. Secondly, we can identify and choose a proper extension-model with the characteristic of points $\left\{x_{i}, y_{i}\right\}$. Thirdly, we should determine whether the feature points meet the extension-model chosen. If not, we should reselect some new feature points from the curve again. Finally, the curve can be extended according to the final feature points and the extension-model chosen.

\subsection{Applications of Accurate Intelligent Extension}

As Table 1 shows, four groups of feature points on the four curves can describe the characteristics of those curves, respectively. The four curves to be extended are as follows: quadratic curve, cubic curve, exponential curve, and Gompertz curve. We will extend the four curves using the above method, and analyze the errors between extended curves and theoretical curves, to test the accuracy of the four extensionmodels. 


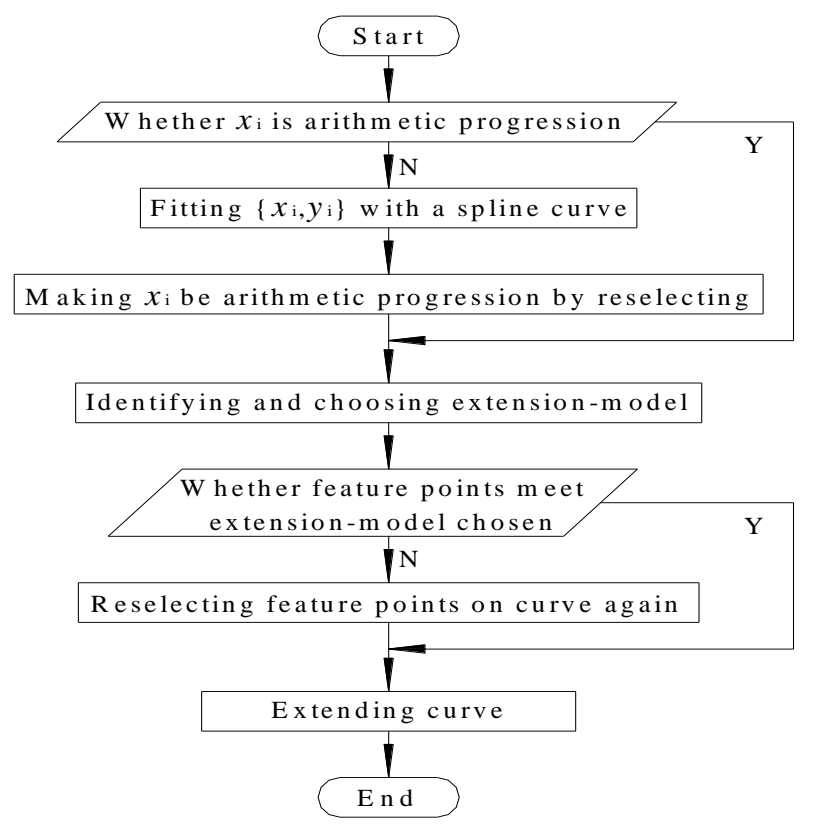

Figure 1. Flow Chart for Intelligent Extension

Table 1. Feature Points and Curvilinear Equations for Accurate Intelligent Extension

\begin{tabular}{|c|c|c|}
\hline No. & Feature points & $\begin{array}{l}\text { Curvilinear } \\
\text { equations }\end{array}$ \\
\hline 1 & $\begin{array}{c}(1.000,6.000) ;(1.500,10.750) ;(2.400,23.080) ;(3.000 \\
34.000) ;(5.000,86.000) ;(6.300,132.670)\end{array}$ & $\begin{array}{l}y=3 x^{2}+2 x+1 \\
1.000 \leq x \leq 6.300\end{array}$ \\
\hline 2 & $\begin{array}{c}(2.000,49.000) ;(3.000,142.000) ;(3.500,155.194) ;(3.800 \\
271.408) ;(4.100,335.314) ;(5.000,586.000)\end{array}$ & $\begin{array}{c}y=4 x^{3}+3 x^{2}+2 \\
x+1 \\
2.000 \leq x \leq 5.000\end{array}$ \\
\hline 3 & $(1.000,22.167) ;(1.500,60.257) ;(2.000,163.795) ;(2.500,445.240)$ & $\begin{aligned} y & =3 e^{2 x} \\
1.000 & \leq x \leq 2.500\end{aligned}$ \\
\hline 4 & $\begin{array}{c}(1.000,1.399) ;(1.100,1.416) ;(1.300,1.450) ;(1.500,1.483) \\
(1.900,1.543) ;(2.000,1.557) ;(2.100,1.571) ;(2.300 \\
1.597) ;(2.400,1.610)\end{array}$ & $\begin{array}{c}y=2 \times 0.6^{0.7^{x}} \\
1.000 \leq x \leq 2.400\end{array}$ \\
\hline
\end{tabular}

Because of the coordinate values $x_{i}$ of the four groups of feature points are not arithmetic progressions, we fit the feature points $\left\{x_{i}, y_{i}\right\}$ with some spline curves respectively at first, and then reselect several new feature points on those curves to make $x_{i}$ be arithmetic progressions. The applied conditions of extension-models about four groups of new feature points are calculated respectively, as shown in Table 2. The maximum difference of $\nabla^{2} y_{i}$ of curve 1 is minimum, is nearly constant. Thus, the quadratic extension-model is adopted for curve 1 . The maximum difference of $\nabla^{3} y_{i}$ of curve 2 is minimum, is nearly constant, and then the cubic extension-model is adopted 
for it. The maximum difference of $\nabla \ln y_{i}$ of curve 3 is minimum, is nearly constant, and then the exponential extension-model is adopted for it. The maximum difference of $\left(\nabla \ln y_{i+1}-\nabla \ln y_{i}\right) / \nabla \ln y_{i}$ of curve 4 is minimum, is nearly constant, and then the Gompertz extension-model is adopted for it.

Table 2. Choosing Intelligent Extension-Models

\begin{tabular}{|c|c|c|c|c|}
\hline \multirow{2}{*}{ No. } & Quadratic curve & Cubic curve & Exponential curve & Gompertz curve \\
\cline { 2 - 5 } & $\nabla^{2} y_{i}$ & $\nabla^{3} y_{i}$ & $\nabla \ln y_{i}$ & $\left(\nabla \ln y_{i+1}-\nabla \ln y_{i}\right) / \nabla \ln y_{i}$ \\
\hline 1 & $5.116 \times 10^{-14}$ & $9.095 \times 10^{-14}$ & $1.140 \times 10^{-2}$ & $4.800 \times 10^{-3}$ \\
\hline 2 & $4.700 \times 10^{-3}$ & $6.821 \times 10^{-13}$ & $5.700 \times 10^{-3}$ & $1.600 \times 10^{-3}$ \\
\hline 3 & 25.387 & 0.503 & $2.447 \times 10^{-15}$ & $2.220 \times 10^{-13}$ \\
\hline 4 & $9.869 \times 10^{-7}$ & $5.388 \times 10^{-9}$ & $6.454 \times 10^{-4}$ & $8.590 \times 10^{-13}$ \\
\hline
\end{tabular}

For curve 1, the undetermined parameters by calculating are as follows: $\hat{a}=3.000$ $3.271 \times 10^{-13}, \hat{b}=2.000+1.430 \times 10^{-10}, \hat{c}=1.000-5.720 \times 10^{-11}$. We can extend the curve with the model (3) from the starting point $(6.300,132.670)$. Its extending plots and error plots with the theoretical curve are shown as Figure 2. It is shown that the error is of the order of magnitude of $10^{-11}$, and its precision is superior.
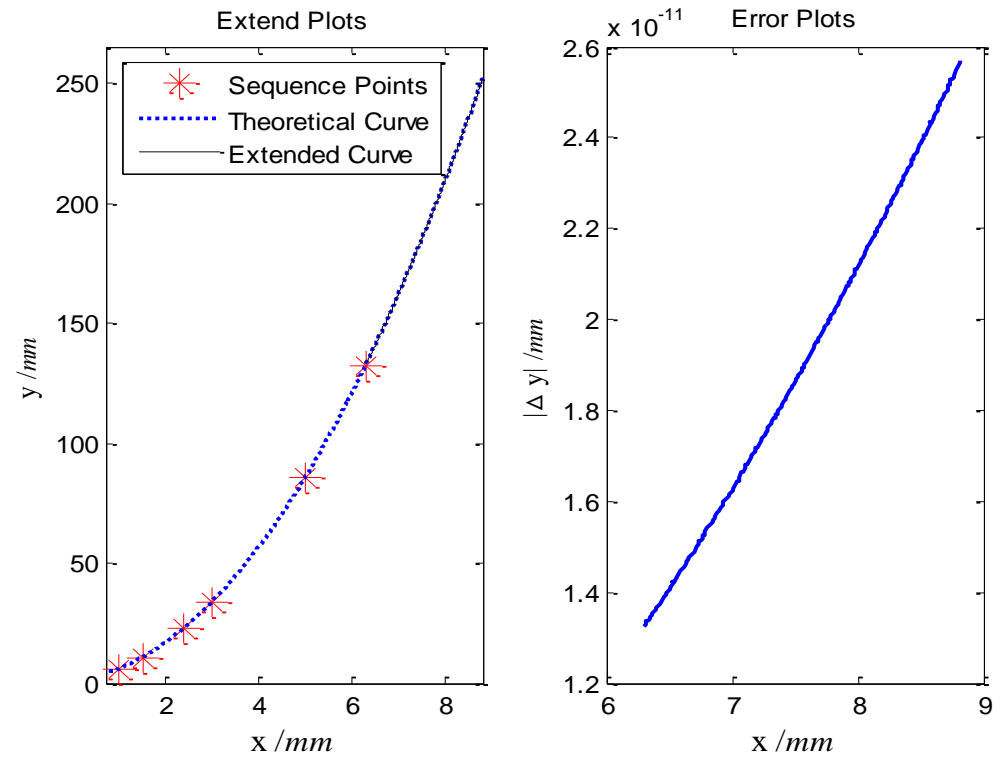

Figure 2. Extending Plots \& Error Plots of Curve 1

For curve 2, the undetermined parameters by calculating are as follows: $\hat{a}=$ $4.000+5.132 \times 10^{-14}, \hat{b}=3.000-7.621 \times 10^{-13}, \hat{c}=2.000-3.291 \times 10^{-11}, \hat{d}=1.000+9.282 \times 10^{-}$ 10. We can extend the curve with the model (10) from the starting point $(5.000$, 586.000). The extending plots and error plots with the theoretical curve are shown as Figure 3. It is shown that the error is of the order of magnitude of 10-5, and its precision is high. 

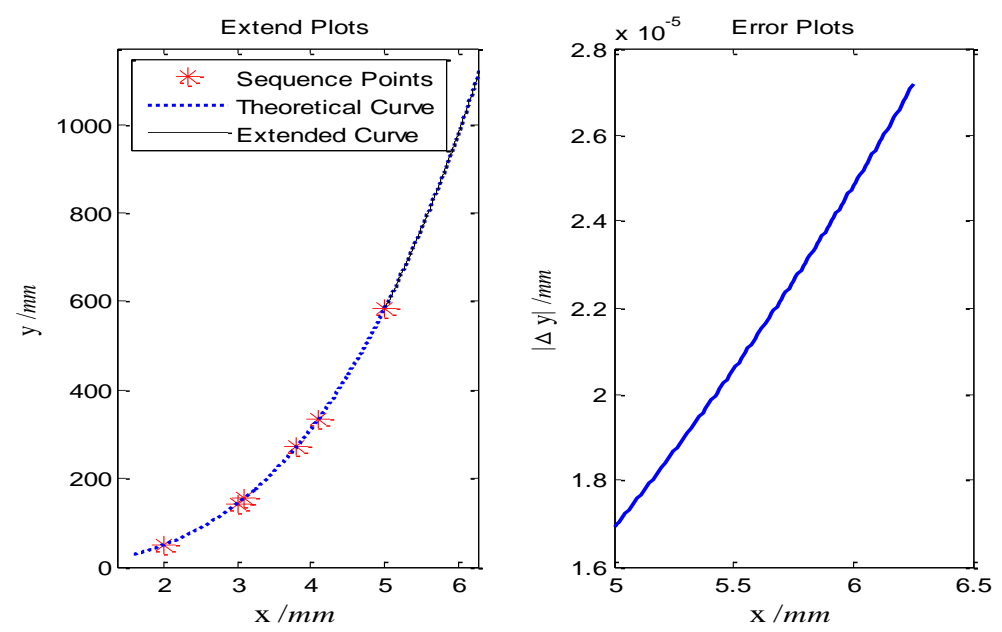

Figure 3. Extending Plots \& Error Plots of Curve 2

For curve 3, the undetermined parameters by calculating are as follows: $\hat{a}=$ $2.000+1.260 \times 10^{-13}, \hat{b}=3.000-3.791 \times 10^{-13}$. We can extend the curve with the model (14) from the starting point $(2.500,445.240)$. Its extending plots and error plots with the theoretical curve are shown as Figure 4. It is shown that the error is of the order of magnitude of $10^{-12}$, and its precision is superior.
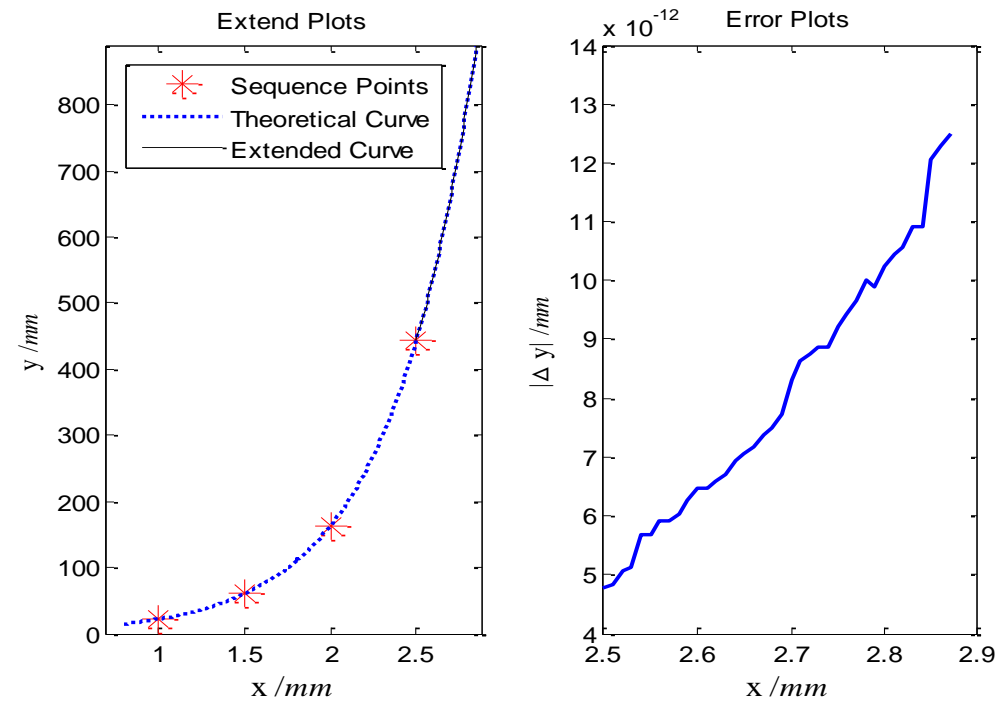

Figure 4. Extending Plots \& Error Plots of Curve 3

For curve 4, the undetermined parameters by calculating are as follows: $\hat{a}=$ $0.600+5.100 \times 10^{-11}, \hat{b}=0.700-2.901 \times 10^{-11}, \hat{k}=2.000+7.830 \times 10^{-10}$. We can extend the curve with the model (25) from the starting point $(2.400,1.610)$. Its extending plots and error plots with the theoretical curve are shown as Figure 5. It is shown that the error is of the order of magnitude of $10^{-11}$, and its precision is superior. 

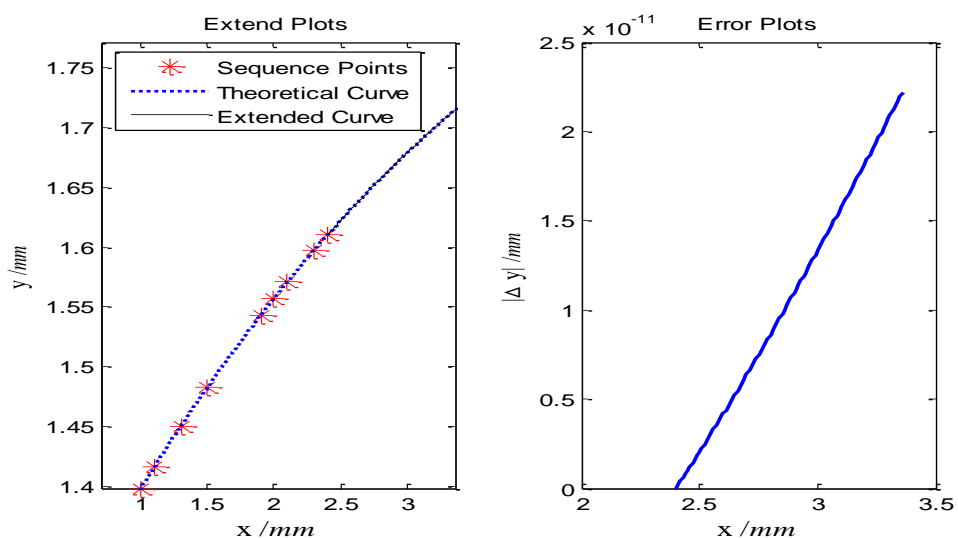

Figure 5. Extending Plots \& Error Plots of Curve 4

\subsection{Applications of Approximate Intelligent Extension}

In most cases, curves to be extended does not belong to one of above four things, usually be non-circular curves with arbitrary shapes. These non-circular curves can also be extended approximately using the aforementioned models in CAD while they are used for connection or transition without high precision [15].

As Table 3 shows, four groups of feature points on four non-circular curves can describe the characteristics of those curves, respectively. These curvilinear equations are different from the extension-models. We will extend the four curves using the above method, and analyze the errors between extended curves and theoretical curves, to test the effectiveness of the approximate intelligent extension.

\section{Table 3. Feature Points and Curvilinear Equations for Approximate Intelligent Extension}

\begin{tabular}{|c|c|c|}
\hline No. & Feature points & $\begin{array}{c}\text { Curvilinear } \\
\text { equations }\end{array}$ \\
\hline I & $\begin{array}{c}(-11.673,-20.752) ;(-16.341,-18.920) ;(-20.815,-16.102) ;(-24.910,- \\
12.292) ;(-28.435,-7.516) ;(-31.197,-1.824) ;(-33.000,4.704)\end{array}$ & $\begin{array}{l}\left\{\begin{array}{l}x=r \cos \theta / \theta \\
y=r \sin \theta / \theta\end{array}\right. \\
1.090 \leq \theta \leq 4.390\end{array}$ \\
\hline$\Pi$ & $\begin{array}{c}(1.000,0.842) ;(3.000,0.423) ;(4.000,-3.027) ;(4.500,-4.399) ;(4.800,- \\
4.782)\end{array}$ & $\begin{array}{c}y=x \sin x \\
1.000 \leq x \leq 4.800\end{array}$ \\
\hline III & $\begin{array}{c}(-0.198,0.140) ;(-0.172,0.143) ;(-0.118,0.153) ;(-0.034,0.176) ;(0.057 \\
0.216) ;(0.166,0.274) ;(0.281,0.351)\end{array}$ & $\left\{\begin{array}{l}x=r(\cos \theta)^{3}+k_{1} \\
y=r(\sin \theta)^{3}+k_{2} \\
-0.198 \leq x \leq 0.281\end{array}\right.$ \\
\hline IV & $\begin{array}{c}(1.000,8.443) ;(1.200,7.561) ;(1.400,6.868) ;(1.600,6.317) ;(1.800 \\
5.874) ;(2.000,5.513) ;(2.200,5.217)\end{array}$ & $\begin{array}{c}y=k_{1} / x+k_{2} \\
1.000 \leq x \leq 2.200\end{array}$ \\
\hline
\end{tabular}

For the sake of the coordinate values $x_{i}$ of the four groups of feature points are not arithmetic progressions, we fit them with spline curves respectively at first, and then reselect several new feature points from the curves to make $x_{i}$ be arithmetic progressions. The applied conditions of extension-models about four groups of new feature points are calculated 
respectively, as shown in Table 4. The maximum difference of $\nabla^{2} y_{i}$ of curve I is minimum. Thus, the quadratic extension-model is adopted for curve I . The maximum difference of $\nabla^{3} y_{i}$ of curve $\Pi$ is minimum, and then the cubic extension-model is adopted for it. The maximum difference of $\nabla \ln y_{i}$ of curve $\mathrm{III}$ is minimum, and then the exponential extension-model is adopted for it. The maximum difference of $\left(\nabla \ln y_{i+1}-\nabla \ln y_{i}\right) / \nabla \ln y_{i}$ of curve IV is minimum, and then the Gompertz extension-model is adopted for it.

Table 4. Choosing Approximate Intelligent Extension-Models

\begin{tabular}{|c|c|c|c|c|}
\hline \multirow{2}{*}{ No. } & Quadratic curve & Cubic curve & Exponential curve & Gompertz curve \\
\cline { 2 - 5 } & $\nabla^{2} y_{i}$ & $\nabla^{3} y_{i}$ & $\nabla \ln y_{i}$ & $\left(\nabla \ln y_{i+1}-\nabla \ln y_{i}\right) / \nabla \ln y_{i}$ \\
\hline I & $7.840 \times 10^{-4}$ & $1.170 \times 10^{-2}$ & 0.082 & 0.008 \\
\hline II & 0.001 & $1.760 \times 10^{-5}$ & 8.214 & 5.518 \\
\hline III & $1.003 \times 10^{-5}$ & 0.002 & $7.096 \times 10^{-8}$ & 0.003 \\
\hline IV & 0.067 & 1.250 & 8.214 & $7.369 \times 10^{-4}$ \\
\hline
\end{tabular}

For curve I, $r=100.000$, the undetermined parameters by calculating are as follows: $\hat{a}$ $=0.013, \hat{b}=-2.038, \hat{c}=20.198$. We can extend the curve with the model (3) from the starting point $(-33.000,4.704)$. Because of the symmetric axis and the vertex of the model (3) are not inconsistent with those of the curve to be extended, we should implement affine transformation for the model (3) to make them coincide, and then extend the curve. The extending plots and error plots with the theoretical curve are shown as Figure 6. It is shown that the curve extended near the starting point is proving well by comparison with the theoretical curve $(x<15.000)$. While the part of the curve extended is far from the starting point, the error becomes larger and cannot be neglected in most cases.

For curve $I$, the undetermined parameters by calculating are as follows: $\hat{a}=0.477, \hat{b}=-$ 4.908, $\hat{c}=13.224, \hat{d}=-7.952$. We can extend the curve with the model (10) from the starting point $(4.800,-4.782)$. The extending plots and error plots with the theoretical curve are shown as Figure 7. It is shown that the curve extended near the starting point is proving well by comparison with the theoretical curve $(x<6.700)$. Farther from the start point larger is the error.

For curve III, $r=1.000, k_{1}=0.800$ and $k_{2}=0.140$. The undetermined parameters by calculating are as follows: $\hat{a}=2.029, \hat{b}=0.211$. We can extend the curve with the model (14) from the starting point $(0.281,0.351)$. The extending plots and error plots with the theoretical curve are shown as Figure 8. It is shown that the curve extended near the starting point is proving well by comparison with the theoretical curve $(x<0.750)$. While the part of the curve extended is far from the starting point, the error becomes larger and cannot be neglected.

For curve IV $k_{1}=5.500$ and $k_{2}=2.700$, the undetermined parameters by calculating are as follows: $\hat{a}=5.498, \hat{b}=0.492$ and $\hat{k}=3.602$. We can extend the curve with the model (25) from the starting point $(2.200,5.217)$. Its extending plots and error plots with the theoretical curve are shown as Figure 9. It is shown that the curve extended near the starting point is proving well by comparison with the theoretical curve $(x<7.000)$. Farther from the start point larger is the error. 

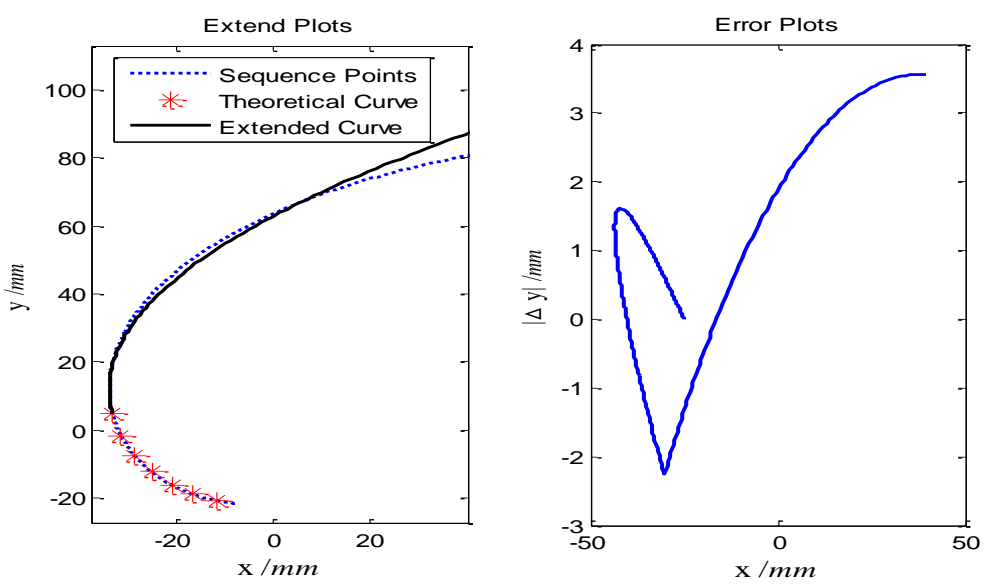

Figure 6. Extending Plots \& Error Plots of Curve I
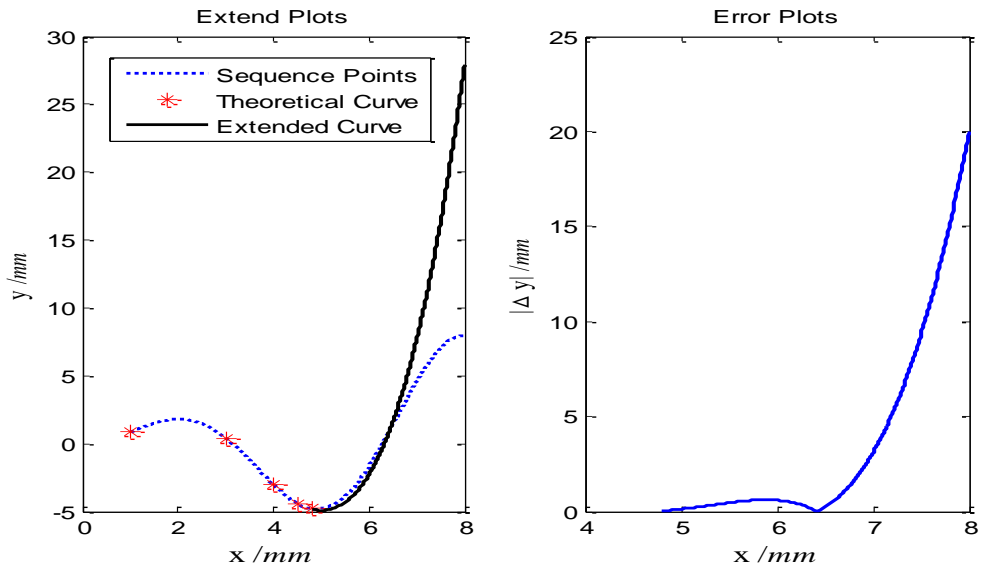

Figure 7. Extending Plots \& Error Plots of Curve II
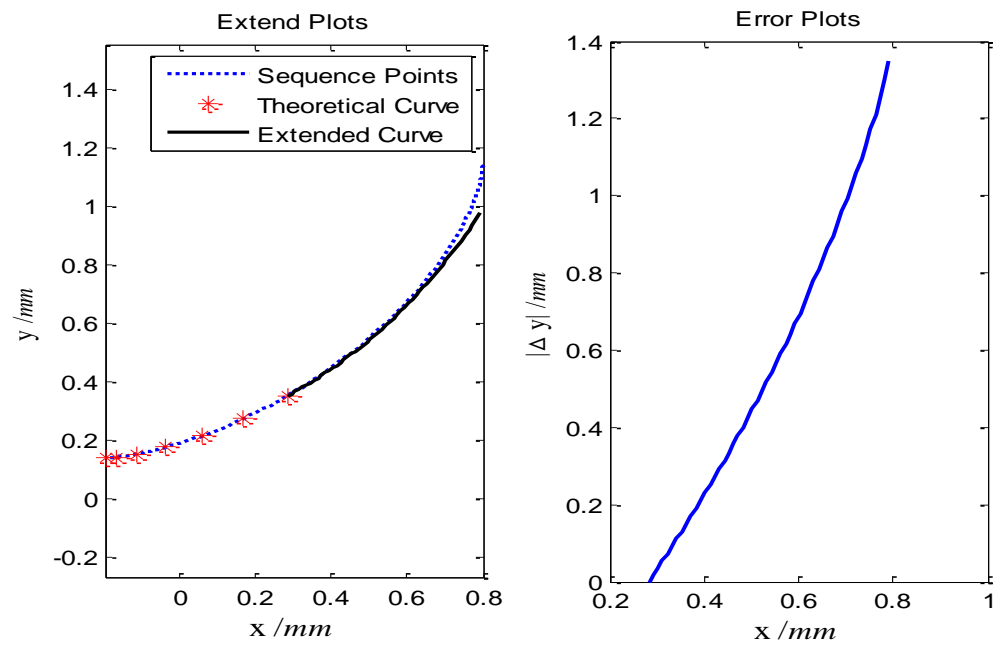

Figure 8. Extending Plots \& Error Plots of Curve III 

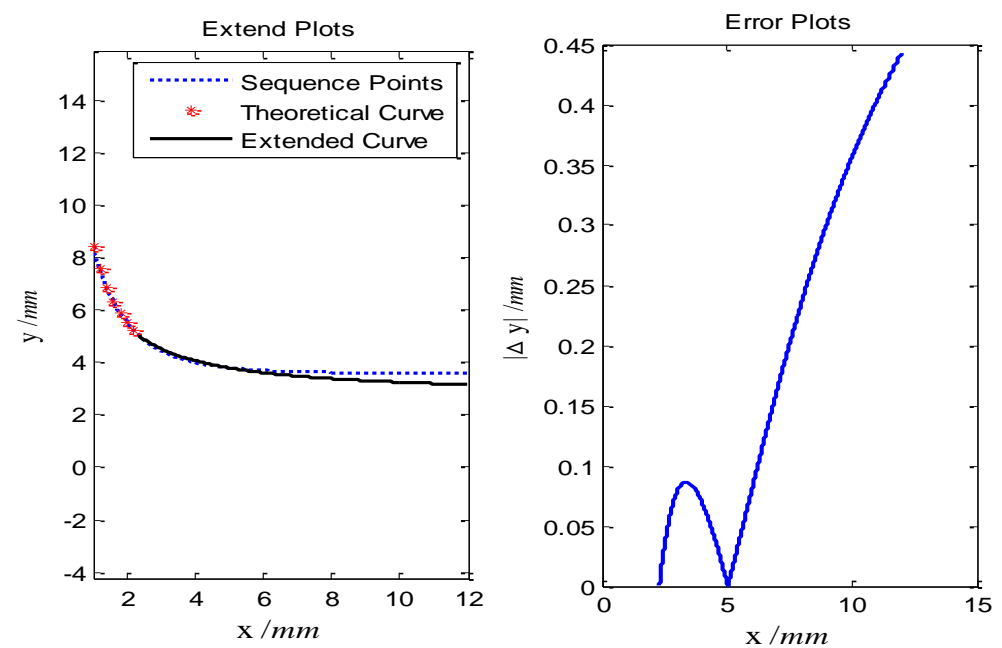

Figure 9. Extending Plots \& Error Plots of Curve IV

\section{Conclusions}

(1) Based on the principles of trend extrapolation, multinomial extension-models (quadratic extension-model and cubic extension-model), exponential extension-model and Gompertz extension-model have been developed, which can be selected and used reasonably according to feature points of non-circular curves. Thus, non-circular curves can be extended intelligently.

(2) Applications of intelligent extension for curves show that curves can be extended for a long distance accurately while they are consistent with the type of extension-models. The accuracy of curves extended is very high. The closer the distance from starting point, the higher the accuracy is. That is to say, an accurate intelligent extension can be achieved under certain conditions. On the other hand, the extension-models can be available for approximate extension for a short distance if the types of the non-circular curves are different from theirs, with arbitrary shapes. The curves extended near starting points are proving well by comparison with theoretical curves. While the part of curves extended is far from the starting point, the error becomes larger and cannot be neglected in most cases. To achieve accurate intelligent extension for them, it is possible to develop other extension-models for those noncircular curves with corresponding shapes.

(3) Some core technologies and key generic problems that non-circular curves will be extended in universal CAD softwares are solved, which can be used for existing CAD softwares to upgrade or develop secondarily, and then increase or improve their extension functions for non-circular curves.

\section{Acknowledgement}

This work was supported by the Natural Science Foundation of Anhui Province of China (1408085ME94) and the Natural Science Research Project of Higher Education of Anhui Province of China (KJ2013A039).

\section{References}

[1] S. K. Smith, J. Tayman and Swanson, "The Springer Series on Demographic Methods and Population Analysis. Springer", (2002); Berlin, Germany. 
[2] G. Laurent, "Analysis and short-time extrapolation of stock market indexes through projection onto discrete wavelet subspaces", Nonlinear Analysis: Real World Applications, (2010), pp. 3139-3154.

[3] H. Tim, D. Damian, D. Zoe and L. Alan, "Reconstruction of long-term tobacco consumption trends in Australia and their relationship to lung cancer mortality", Cancer Causes and Control, (2011), pp. 1047-1053.

[4] G. Hugues, L. Wouter, D. M. Anne, C. Elisabeth and H. O. Alejandro, "Consistent past half-century trends in the atmosphere, the sea ice and the ocean at high southern latitudes", Climate Dynamics, (2009), pp. 9991016.

[5] Y. Y. Liu, J. Han and G. Z. Zhang, "Research on Intelligent Extension Technology for Curves Based on Trend Extrapolation", Advanced Materials Research, (2012), pp. 2596-2599.

[6] T. K. Naskar and R. Mishra, "Introduction of control points in B-splines for synthesis of ping finite optimized cam motion program”, Journal of Mechanical Science and Technology, (2012), pp. 489-494.

[7] D. L. Vladimir, "Grid Generation Methods: Scientific Computation", (2010); London, England.

[8] X. Liu, Y. B. Zhang, B. O. Zheng and H. Shi, "Periodic and subharmonic solutions for second orderLaplacian difference equations", Proceedings Mathematical Sciences, (2011), pp. 457-468.

[9] A. M. Yazdani, A. Ahmadi, S. Buyamin, M. F. Rahmat, F. Davoudifar and H. A. Rahim, "Imperialist Competitive Algorithm-based Fuzzy PID Control Methodology for Speed Tracking Enhancement of Stepper Motor", The International Journal on Smart Sensing and Intelligent Systems, (2012), pp. 717-741.

[10] N. Parhi and A. Panda, "Asymptotic approximation of solutions of nonlinear third order difference equations", Mathematica Slovaca, (2011), pp. 39-54.

[11] B. Albert, "On Exponential Growth and Half-Lives: A Comment on Bermingham", Population \& Environment, (2003), pp. 61-69.

[12] E. M. Drakakis and A. J. Payne, "A Bernoulli Cell-Based Investigation of the Non-Linear Dynamics in LogDomain Structures”, Analog Integrated Circuits and Signal Processing, (2000), pp. 127-146.

[13] N. J. Moura and M. B. Ribeiro, "Evidence for the Gompertz curve in the income distribution of Brazil 19782005", The European Physical Journal B - Condensed Matter and Complex Systems, (2009), pp. 101-120.

[14] H. A. Rahim, F. Ibrahim and M. N. Taib, "System Identification of Nonlinear Autoregressive Models in Monitoring Dengue Infection", The International Journal on Smart Sensing and Intelligent Systems, (2010), pp. 783-805.

[15] J. D. Foley and R. V. Dam, "Introduction to computer graphics. Pearson Education", (2003); New York, USA.

\section{Author}

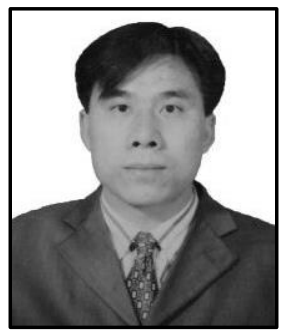

Youyu Liu is a researcher at Anhui Polytechnic University, and a $\mathrm{PhD}$ Candidate at Hefei University of Technology. He received BE and ME degrees in Lanzhou University of Technology in Lanzhou, China. His research interest is computer aided design. He has been an Associate Professor since 2012, and published more than 40 papers. 